\title{
Identidades que importan. Trans e intersex, la ley argentina y la irrupción de la ciencia
}

\section{[Identities that Matter. Trans and Intersex, the Argentinian Law, and the Irruption of Science]}

\author{
MARIANA CÓRDOBA \\ Consejo Nacional de Investigaciones Científicas y Técnicas \\ Universidad de Buenos Aires \\ marianacordoba@conicet.gov.ar
}

\begin{abstract}
Resumen: Como resultado de las luchas de las disidencias sexogenéricas se han aprobado leyes en todo el mundo que garantizan el reconocimiento de las identidades generizadas. La Ley de Identidad de Género argentina (2012) ofrece una definición de "identidad de género" opuesta a los enfoques biológicos y a la patologización y que respeta la voluntad individual y niega todo lugar a los refrendos de terceros "autorizados". Esta ley regula el acceso a prácticas médicas para intervenir libremente los cuerpos. Ahora bien, los principios científicos del dimorfismo sexual sobre los cuales descansan esos procedimientos suelen denunciarse por reprimir, normativizar y patologizar en especial los cuerpos intersex. Analizaré la cuestión de las identidades de género a la luz de esta tensión; por último, argumentaré que la identidad es transformativa, relacional y biotecnológicamente producida.
\end{abstract}

Palabras clave: desbiologización, ley de identidad de género, identidad como transformación, identidad relacional, identidad biotecnológicamente producida

\begin{abstract}
As a result of the struggles of sex and gender dissidents, laws that guarantee the recognition of gender-identities have been passed all around the world. The Argentinian Gender Identity Law (2012) offers a definition of "gender identity" as opposed to biological anchorages and pathologization, respecting personal will and leaving no room for endorsement by "authorised" third parties. This law regulates the access to medical practices to freely intervene bodies. However, the theoretical scientific principles of sexual dimorphism on which these procedures are based on are usually denounced for repressing, normalizing and pathologizing especially intersex bodies. I will analyse the issue of gender identities in the light of this tension and, finally, I will argue that identity is transformative, relational and biotechnologically produced.

Key words: de-biologization, gender identity law, identity as transformation, relational identity, biotechnologically produced identity
\end{abstract}

\section{Introducción}

Tanto la cuestión de la identidad de las personas como la de la identidad de colectivos y comunidades ha sido abordada desde muy diversas 
perspectivas teóricas y de compromisos políticos también muy diversos. El problema de la identidad, que supo fascinar a filósofos de diferentes corrientes del pensamiento en la historia de la filosofía, devino cada vez menos un problema teórico y cada vez más una cuestión práctica, con lo que dio lugar a debates intensos en torno a las políticas de la identidad (cfr. Heyes 2018). La propia noción de identidad ha sido objeto de numerosas críticas que han llevado a propugnar incluso su completo abandono.

Muchas inquietudes recorrieron ese debate: ¿debe basarse la identidad en un rasgo esencial? Si no se la hace descansar en una esencia ¿tiene sentido la noción de identidad? En la medida en que excluye a otr*s sujet*s, ¿cualquier categorización implica necesariamente violencia? ¿Toda apelación a la identidad es conservadora y deudora de una exaltación de la subjetividad, la individualidad y la autenticidad? ¿Por qué no dejamos de hablar de identidad?

Desde esta perspectiva, en el presente trabajo me propongo analizar la cuestión de las identidades de género y, en especial, la cuestión de las identidades trans, ${ }^{1}$ para recuperar el problema filosófico de la identidad y algunas líneas fundamentales del debate sobre las políticas de la identidad en ciertos enfoques feministas y de los estudios de género.

Para analizar la cuestión de las identidades de género e identidades trans, me centraré en la Ley de Identidad de Género (Ley 26.743) promulgada en Argentina en el año 2012. Consideraré el papel del conocimiento científico que está en la base de las prácticas biomédicas a las que es posible acudir para modificar los cuerpos según la identidad de género autopercibida - que es el mismo conocimiento en que se basan las intervenciones a los cuerpos intersex- y cuyo acceso precisamente regula esa ley.

En primer término, este trabajo tiene un objetivo programático: busco proponer una tarea para la filosofía de la ciencia. En segundo lugar, el objetivo fundamental es avanzar hacia la configuración de una noción filosófica propia de identidad que atienda el punto de vista de las comunidades trans y que contribuya a pensar políticas transformativas para la ampliación de derechos y reconocimiento de todas las personas.

${ }^{1}$ Dado que me referiré a la Ley de Identidad de Género en Argentina, incluyo en la categoría de "trans" a la comunidad travesti, transexual y transgénero argentina. Mi inclusión de tales personas (de carne y hueso, o la inclusión posible de otras personas) en una categoría específica se basa en particular en su forma de autodenominarse y autoidentificarse como "trans". Respecto del propio punto de vista de las comunidades, véase el informe nacional sobre la comunidad argentina en Berkins 2015. 
Argumentaré, con base en las ideas de Bornstein 1994, que la identidad de género es transformativa y, siguiendo a Heyes 2003, que es relacional. Asimismo, propondré que la identidad de género es biotecnológicamente producida, una idea que contempla el papel productivo del conocimiento científico detrás de las prácticas biomédicas. Argumentaré que la ciencia no sólo corrige, reprime y patologiza, sino que también gestiona y produce identidad.

\section{La ley de identidad de género en Argentina: un avance innegable}

A lo largo de la historia, las personas y comunidades trans han sido depositarias de la fobia y la discriminación sistemática dirigidas en buena parte de manera activa por el Estado (a través de la represión policial y la criminalización de sus identidades por medio de los edictos policiales) y también resultado de la ausencia del Estado al negar reconocimiento legal a sus identidades (Mouratian 2012). Las comunidades han sido - y aún son - objeto de violencia, estigmatización y exclusión (cfr. Berkins y Fernández 2006, Berkins 2015). En el contexto de la lucha de las propias comunidades contra la discriminación y violencia ejercida por el Estado y por la sociedad argentina en general, en la década de los noventa el movimiento trans logró colocar en la agenda pública la discusión sobre la legitimidad de los cuerpos en las calles, lo que representó un primer paso en términos de visibilidad del movimiento (Mouratian 2012). A lo largo de estos años, las "marchas del orgullo" también alcanzaron una mayor visibilidad y el movimiento LGBTTIQ + - con un mayor o menor nivel de organización y repercusión según el periodo- ha ido conquistando espacios en una sociedad ciega a su existencia, cuyas instituciones no producían ningún cambio real. Tuvo que llegar el año 2002 para que la lucha del movimiento produjera un cambio legal importante con la aprobación en la ciudad de Buenos Aires de la Ley de Unión Civil para parejas "con independencia de su sexo o su orientación sexual". Ocho años más tarde se reformó el Código Civil, lo que dio lugar a la Ley de Matrimonio Igualitario (Ley 26.618). Por último, en agosto de 2011 inició la discusión de la Ley de Identidad de Género en el Congreso de la Nación Argentina. Como antecedentes de la ley cabe destacar que, en 2008, la justicia argentina por primera vez autorizó la modificación del documento de identidad de una persona sin que ésta se hubiera realizado intervención médica alguna. Desde el año 2007 se presentaron en el país diversos proyectos de ley para garantizar los derechos humanos de las personas trans -entre ellos, el derecho a la salud y el reconocimiento de las identidades-

Revista de Filosofía Diánoia, vol. 65, no. 84 (mayo-octubre de 2020) e-ISSN: 1870-4913 • DOI: https://doi.org/10.22201/iifs.18704913e.2020.84.1587 
y en el año 2006 se firmó la Carta Acuerdo entre el Hospital Durand, de la Ciudad de Buenos Aires, y la Comunidad Homosexual Argentina (CHA) para crear un equipo interdisciplinario para la atención de la comunidad gay, lésbica, travesti, transexual y bisexual. En noviembre de 2011, la Cámara de Diputados aprobó la Ley de Identidad de Género y lo mismo hizo la Cámara de Senadores en mayo de 2012. El 24 de mayo de ese año se promulgó y publicó en el Boletín Oficial a través del Decreto $773 / 12 .^{2}$

En el artículo $1^{\circ}$, la ley establece el derecho a la identidad de género. Afirma que toda persona tiene derecho "al reconocimiento de su identidad de género; al libre desarrollo de su persona conforme a su identidad de género; a ser tratada de acuerdo con su identidad de género $y$, en particular, a ser identificada de ese modo en los instrumentos que acreditan su identidad respecto de el/los nombre/s de pila, imagen y sexo con los que allí es registrada". En el artículo $2^{\circ}$, la Ley ofrece una definición de "identidad de género": "Se entiende por identidad de género a la vivencia interna e individual del género tal como cada persona la siente, la cual puede corresponder o no con el sexo asignado al momento del nacimiento, incluyendo la vivencia personal del cuerpo". Luego de la definición, en este mismo artículo se establece la posibilidad, por la libre elección de cada persona, de modificar el propio cuerpo de acuerdo con la identidad de género autopercibida. El texto señala: "Esto puede involucrar la modificación de la apariencia o la función corporal a través de medios farmacológicos, quirúrgicos o de otra índole, siempre que ello sea libremente escogido. También incluye otras expresiones de género, como la vestimenta, el modo de hablar y los modales". Y en su artículo $3^{\circ}$ establece que "Toda persona podrá solicitar la rectificación registral del sexo, y el cambio de nombre de pila e imagen, cuando no coincidan con su identidad de género autopercibida". En el artículo 11, "Derecho al libre desarrollo personal", se establece el derecho para toda persona mayor de edad de acceder "a intervenciones quirúrgicas totales y parciales y/o tratamientos integrales hormonales para adecuar su cuerpo, incluida su genitalidad, a su identidad de género autopercibida, sin necesidad de requerir autorización judicial o administrativa". Asimismo, se hace explícita la independencia en el acceso, por un lado, a los tratamientos hormonales y, por otro, a los procedimientos quirúrgicos: "Para el acceso a los tratamientos integrales hormonales, no será necesario acreditar la voluntad

${ }^{2}<$ http://servicios.infoleg.gob.ar/infolegInternet/anexos/195000-199999/197 860/norma.htm>, consultado el 16/11/2018.

Revista de Filosofía Diánoia, vol. 65, no. 84 (mayo-octubre de 2020) e-ISSN: 1870-4913 • DOI: https://doi.org/10.22201/iifs.18704913e.2020.84.1587 
en la intervención quirúrgica de reasignación genital total o parcial. En ambos casos se requerirá, únicamente, el consentimiento informado de la persona". ${ }^{3}$ Por último, este artículo obliga a los prestadores de servicios médicos a brindar los tratamientos y procedimientos quirúrgicos a los que se refiere la ley:

Los efectores del sistema público de salud, ya sean estatales, privados o del subsistema de obras sociales, deberán garantizar en forma permanente los derechos que esta ley reconoce. Todas las prestaciones de salud contempladas en el presente artículo quedan incluidas en el Plan Médico Obligatorio, o el que lo reemplace, conforme lo reglamente la autoridad de aplicación.

Por su parte, el artículo 12 establece el derecho al trato digno que debe recibir toda persona:

Deberá respetarse la identidad de género adoptada por las personas, en especial por niñas, niños y adolescentes, que utilicen un nombre de pila distinto al consignado en su documento nacional de identidad. A su solo requerimiento, el nombre de pila adoptado deberá ser utilizado para la citación, registro, legajo, llamado y cualquier otra gestión o servicio, tanto en los ámbitos públicos como privados. [...] En aquellas circunstancias en que la persona deba ser nombrada en público deberá utilizarse únicamente el nombre de pila de elección que respete la identidad de género adoptada.

Aún existen en el país numerosos límites para la aplicación de la Ley de Identidad de Género porque, por ejemplo, muchos hospitales públicos no cuentan con los recursos ni los profesionales con la capacitación necesaria para realizar los procedimientos requeridos. Además, el hecho de que la ley obligue a reconocer la identidad de género de todas las personas no es suficiente para erradicar la discriminación y la violencia de una sociedad que no está todavía a la altura de la ley. A pesar

${ }^{3}$ Para los menores de edad, el mismo artículo establece: "En el caso de las personas menores de edad regirán los principios y requisitos establecidos en el artículo $5^{\circ}$ para la obtención del consentimiento informado. Sin perjuicio de ello, para el caso de la obtención del mismo respecto de la intervención quirúrgica total o parcial se deberá contar, además, con la conformidad de la autoridad judicial competente de cada jurisdicción, quien deberá velar por los principios de capacidad progresiva e interés superior del niño o niña de acuerdo con lo estipulado por la Convención sobre los Derechos del Niño y en la Ley 26.061 de protección integral de los derechos de las niñas, niños y adolescentes". No me detendré en la especificidad de la reglamentación para menores porque excede los propósitos del trabajo.

Revista de Filosofía Diánoia, vol. 65, no. 84 (mayo-octubre de 2020) e-ISSN: 1870-4913 • DOI: https://doi.org/10.22201/iifs.18704913e.2020.84.1587 
de esto, como sostiene Diana Maffia, la ley constituye un avance legal indiscutible, y es "la mejor del mundo que avanza sobre derechos que se están procurando a nivel internacional" (Maffia 2015). Sin duda, nuestro país "está a la vanguardia en las leyes de igualdad de derechos a nivel mundial, es junto a Dinamarca el único país que sólo exige la expresión de voluntad de la persona para lograr el cambio de nombre [de acuerdo con] su identidad de género autopercibida" (INADI 2016).

En efecto, la ley es revolucionaria por muchas razones y en múltiples aspectos de su texto. En primer lugar, analicemos a quiénes se dirige y a qué obliga. La ley es fruto de la lucha política de las comunidades trans, en cuyo seno fue promovida y discutida y cuyos derechos reconoce, pero establece un derecho para todas las personas, no sólo para las personas trans. Que las personas cisgénero puedan dar por sentado el derecho a sus propias identidades de género es un hecho que justo la ley trae a la luz. Al reglamentar para la Argentina un derecho universal que los Estados deben cumplir, la ley es también ocasión para explicitar que ciertos derechos universales son, de hecho, diferenciales: para ciertos grupos la vulneración sistemática de esos derechos es una marca esencial. De acuerdo con los Principios de Yogyakarta, ${ }^{4}$ si bien los derechos humanos son universales y se ha avanzado en el sentido de que toda persona, sea cual fuere su orientación sexual o identidad de género, goce de la misma dignidad y respeto que cualquier otra, en el mundo sobreabundan las violaciones a los derechos humanos sobre la base de la orientación sexual e identidad de género. Así, las identidades cisgénero configuran un universo de personas con derecho pleno al respeto y reconocimiento de sus identidades, las que son respetadas sin más -así como es respetada sin más la orientación sexual de las personas heterosexuales-.

Tanto las costumbres arraigadas en distintas sociedades como ciertas leyes (relativas, por ejemplo, a los nombres y al sexo que debe consignarse en documentos de identidad) dificultan el reconocimiento pleno de las identidades trans. Operan como vigilantes de las disidencias, al señalarlas de manera permanente. Las prácticas violentas, como el transodio y la transfobia —que suelen encarnarse en los más brutales asesinatos de travestis- muestran hasta qué punto los derechos humanos de las personas trans son permanentemente violados en distintas sociedades. En mayor o menor medida, estas prácticas obstruyen el reconocimiento de las identidades trans, constituyen violaciones al

\footnotetext{
${ }^{4}$ Cfr. <http://yogyakartaprinciples.org/wp-content/uploads/2016/08/principl es_sp.pdf $>$. Consultado el 16/11/2018.
}

Revista de Filosofía Diánoia, vol. 65, no. 84 (mayo-octubre de 2020)

e-ISSN: 1870-4913 • DOI: https://doi.org/10.22201/iifs.18704913e.2020.84.1587 
derecho al reconocimiento y a la vida digna. Los Principios de Yogyakarta también afirman que la respuesta internacional que se ofrece a tales violaciones de los derechos humanos no son consistentes ni están unificadas.

En Argentina es obligatorio registrar en los documentos de identificación personales (Partida de Nacimiento y Documento Nacional de Identidad, DNI) un "sexo" de dos posibles, masculino o femenino, de acuerdo con la Ley del Nombre (Ley 18.248) (cfr. Cabral 2015). La Ley de Identidad de Género tiene, pues, una importancia innegable para aquellas personas cuya identidad de género no concuerda con la identidad asignada en su documentación al momento de nacer. Pero la ley no obliga a nada a estas personas, sino que obliga al resto de las personas al reconocimiento de la identidad de género y al trato digno.

En segundo lugar, cuando afirmo que la ley no obliga a nada a las personas cuya autopercepción de género es distinta de la registral, me refiero al hecho de que, a diferencia de lo que ocurre en la legislación de otros países, la ley no exige ninguna condición para el reconocimiento de la identidad de género: no es forzoso modificar el DNI ni intervenir médicamente los cuerpos de ninguna manera. El cambio de DNI y el acceso a los tratamientos hormonales y procedimientos quirúrgicos son opcionales; es suficiente el deseo propio, la propia voluntad basada en la vivencia personal para la determinación de la identidad de género que se establece de manera exclusiva en primera persona. La autopercepción que funda la identidad no debe ser demostrada ni refrendada por una tercera persona, es decir, no hay lugar para saberes periciales ni voces de terceros "autorizados", sean profesionales de la salud o funcionarios del poder judicial. Por lo tanto, para el acceso a las prácticas médicas basta el consentimiento informado.

En tercer lugar, la ley establece la independencia entre el cambio de DNI, la utilización de tratamientos hormonales y los procedimientos quirúrgicos. Cualquier persona puede someterse a todas estas modificaciones, o a cualquiera de las tres, sin necesidad de acudir a otra/s.

Por último, la ley recoge uno de los signos destacados de la lucha política de las comunidades trans por el reconocimiento: la desbiologización, que podría sintetizarse en el lema "la biología no es destino". En este sentido, en la ley no hay ninguna referencia a una contraposición entre un supuesto sexo biológico - como una suerte de condición "natural"- y un género que se caracterice como cultural, psicológico, autopercibido. El texto distingue entre el "sexo asignado al momento del nacimiento" y "la identidad de género autopercibida". No hay referencias a la biología ni a entidades que usualmente se consideran 
autoidentificantes, como "hombre" o "mujer". Es decir, no se refiere a entidades que subsisten como tales con independencia de un marco que otorga reconocimiento o registro. No se contrapone el género autopercibido a una entidad fundamental y última que pertenecería a una realidad independiente o autodeterminada, descubierta y nombrada por la biología. En la propia formulación de la ley no hay lugar para determinantes biológicos ni esencialismos científicos: no se menciona ninguna diferencia biológica ni se hace referencia a ninguna relación consonante o disonante entre características biológicas y sexo, o entre características biológicas y género. Estas ausencias son significativas y responden a una indiscutible victoria de los colectivos militantes.

$\mathrm{Al}$ no hacer referencia a definiciones fundadas en la ciencia, podría pensarse que la ley ha logrado delimitar la cuestión de la identidad de género dejando fuera a la ciencia; ésta no parece cumplir ningún papel respecto de la identidad de género. Sin embargo, la ley establece que toda persona tiene derecho a acceder a tratamientos médicos con el fin de modificar el cuerpo, lo que da lugar a una tensión a la que dedicaré la sección siguiente.

\section{2. ¿Sin lugar para la ciencia?}

Entre las prácticas biomédicas a cuyo acceso regula la ley se hallan las intervenciones quirúrgicas - totales o parciales-y/o los tratamientos hormonales. No cabe duda de que, para toda persona que quiera acceder a estas prácticas y que gracias a la ley puede hacerlo de modo seguro y gratuito, dichas prácticas tienen una función emancipadora.

Como se ha señalado, la ley se muestra opuesta a reducir la vida humana a aquello que supuestamente define la ciencia, sino que muestra un más sofisticado antirreduccionismo intercientífico (o interbiológico), pues no sólo no es necesario intervenir los propios cuerpos en modo alguno, sino que además es posible someterse a alguno de los tratamientos disponibles y no a otros. Al determinar que el tratamiento hormonal no debe supeditarse a la modificación genital, la ley expone un importantísimo antirreduccionismo: se niega que ciertas características del cuerpo sean fundamentales para la determinación e identificación de un género y, sobre esa base, que otras se reduzcan a ellas.

Hasta aquí no parece haber lugar para la ciencia o, mejor dicho, parece haber quedado expresamente en su lugar: excluida de las determinaciones identitarias del género. Sin embargo, las prácticas biomédicas que entran en escena, en la medida en que se reglamenta y habilita su acceso, suponen, en sus aplicaciones o en la base de éstas, ciertos prin- 
cipios teóricos, tanto biológicos como bioquímicos. Surge entonces una tensión que la filosofía debe enfrentar. Las luchas por el reconocimiento de la identidad de género sobre la base del deseo personal, así como otras reivindicaciones de los colectivos por las disidencias sexogenéricas, han estado marcadas por la oposición radical a los esencialismos naturalistas, a la supuesta determinación de la biología. Desde una perspectiva aún más general, podría afirmarse, junto con Gabriel Giorgi, que politizar un fenómeno es, ante todo, desnaturalizarlo y desbiologizarlo (Giorgi 2014). Y si bien en este caso la reglamentación respecto de las prácticas médicas cumple una función emancipadora, esas prácticas implican determinado conocimiento científico que depende de ciertos supuestos teóricos que los propios activismos combaten.

En las discusiones teóricas acerca de la identidad de género hay muchas referencias a la ciencia. Se suele discutir el papel de la ciencia - o si es que tiene alguno- respecto de la identidad de género con un debate que continúa hasta nuestros días: la disputa entre el médico John Money y el investigador Milton Diamond. De acuerdo con Money, la identidad de género depende de la sociabilidad y la crianza y no de características biológicas, pero la sociabilidad debe darse en armonía con la presencia de genitales estéticamente "normales": si una persona tiene un pene "de aspecto normal" y es "tratada como un niño", se identificará con la identidad de género masculina, y si es "tratada como una niña" y tiene una vagina de "aspecto normal", con la femenina. Money realizaba operaciones de corrección genital a niñs que presentaban genitales de aspecto ambiguo. Este médico fue mundialmente conocido por "el caso Joan-John", el caso de David Reimer, un bebé —designado como de sexo masculino sin ninguna dificultad- que a los ocho meses perdió su pene en una operación por mala praxis. Ante el desamparo, los padres acudieron a Money, quien recomendó practicar una operación en los genitales del bebé para constituir quirúrgicamente una vagina, con el convencimiento de que, con genitales femeninos y criado como una niña, se identificaría sin problemas con la identidad de género femenina ( $c f r$. Money y Green 1969). David fue operado en 1966; se le retiraron los testículos, se le creó una vagina, se le indicó un tratamiento hormonal y sus padres lo llamaron Brenda. Sin embargo, a pesar de que Money recurrió a este caso como evidencia probatoria de su hipótesis respecto de la identidad de género, David jamás se identificó con el género femenino (para conocer su historia, cfr. Butler 2006). Diamond examinó el caso de David muchos años después y mostró que la reasignación de sexo había constituido un fracaso; además, en contra de Money argumentó que la sexualidad tiene una "base hormonal" 
y la identidad de género tiene una base biológica cromosómica: hay una suerte de núcleo duro, de núcleo central o esencia del género que es biológica (cfr. Diamond y Sigmundsen 1997). De acuerdo con Diamond, "la presencia del cromosoma Y es base suficiente para la presunción de la masculinidad social" (Butler 2006, p. 97). Todo esto provocó la discusión de si existe alguna relación entre la identidad de género y la biología, si el sexo es "natural" y el género es cultural (cfr. Raymond 1979) o si, al igual que el género, el sexo biológico también se instituye socialmente (Butler 1990). Si bien es posible afirmar que la mayoría de los activismos combaten el determinismo biológico, esto no puede generalizarse sin más porque ciertas posiciones exaltan una diferencia sobre la base de determinaciones biológicas (cfr. Flores Bedregal 2003).

También han surgido múltiples discusiones sobre los tratamientos biomédicos para modificar los cuerpos, denominados en muchos países tratamientos de "transición" o de "reasignación de sexo". Por ejemplo, Hausman 1995 ha argumentado en contra de todo tipo de intervención y tratamiento porque considera que las prácticas biomédicas, lejos de contribuir a la ampliación de los derechos y del reconocimiento para las personas trans, convierten el propio fenómeno de la "transexualidad" en algo completamente dependiente de la medicina y de la biotecnología y que justifica las intervenciones. Por otra parte, se suelen denunciar los efectos nocivos de estos tratamientos y los riesgos para la salud que suponen. ${ }^{5}$ También se suele criticar que la ciencia y la medicina adopten el binarismo hombre/mujer sobre la base de las definiciones biológicas del sexo y el dimorfismo sexual (por ejemplo, Fausto-Sterling 2006, Gaudilliére 2004).

${ }^{5}$ Aunque también se afirma en la bibliografía especializada que si los tratamientos se realizan bajo un control médico estricto, no implican riesgos mayores. Esto es relevante porque las personas trans en Argentina por lo común se someten a tratamientos poco seguros, que no realizan médicos profesionales, situación que la Ley de Identidad de Género busca también a revertir. Por otra parte, debe tenerse en cuenta la escasez de estudios sobre la población trans y los efectos de las terapias hormonales. Sobre las posiciones en conflicto respecto de los riesgos de la administración de estrógeno en mujeres trans, véase Khan, Schmidt, Spittal, Goldstein, Smock y Greene 2019. De hecho, allí se afirma no sólo que escasean estudios sobre la población trans, sino también que los médicos clínicos carecen de experiencia en el tratamiento de personas transgénero y que son reticentes a recetar hormonas. La bibliografía actual sobre los riesgos de la administración de testosterona a varones trans y de anti-andrógenos y estrógenos a mujeres trans, arroja resultados si no contradictorios, al menos conflictivos, y la evidencia disponible es insuficiente para afirmar el riesgo cardiovascular asociado a las terapias hormonales (cfr. Defreyne, Van de Bruaene, Rietzschel, Van Schuylenbergh y T'Sjoen 2019). 
Desde un punto de vista foucaultiano, se suele afirmar que las prácticas biomédicas que tienden a la patologización de las personas trans tienen la función de corregir, disciplinar y normalizar los cuerpos. Diana Maffia cuestiona que el poder médico siga siendo el que define lo "normal" y lo "anormal" (Maffia 2013). La medicina detenta el poder de clasificar los cuerpos sobre la base de cualidades como sanos/enfermos, normales/anormales, típicos/anómalos y, en consecuencia, interviene los cuerpos que considera patológicos, lo cual resulta evidente en el tratamiento de la intersexualidad, como se verá más adelante. Las prácticas biomédicas normativizan al "curar" y "corregir" violentamente los cuerpos intersexuales ${ }^{6}$ que, considerados "indescifrables" por la ciencia, son desde muy temprana edad intervenidos médicamente con el fin de volverlos descifrables. ${ }^{7}$ Ahora bien, las intervenciones del biopoder sobre la intersexualidad y los tratamientos quirúrgicos y hormonales que la Ley de Identidad de Género reglamenta se fundan en el mismo conocimiento científico que respalda el supuesto del dimorfismo sexual.

A pesar de todo esto, para el caso de la ley argentina el acceso opcional a las prácticas es una posibilidad que debe acompañarse porque responde a una demanda histórica. Si seguimos a Butler 2006, podemos aseverar que si los tratamientos permiten que las vidas de muchas personas sean más vivibles, su posibilidad debe celebrarse más allá de toda duda. Sin embargo, también debe atenderse el hecho de que las prácticas biomédicas se erigen sobre la base de supuestos teóricos relativos a las definiciones científicas del sexo que deben revisarse. En este sentido, se puede sostener que la ciencia, que parecía haber sido por fin confinada fuera del ámbito del deseo y la autodeterminación de la identidad de género, se inmiscuye en la propia ley. La paradoja que debemos abordar es la de considerar regresiva la intromisión de la

\footnotetext{
${ }^{6}$ Por definición, son "intersexuales" los individuos que presentan caracteres sexuales considerados masculinos y femeninos en grados variables. Según la Sociedad Intersex de Norteamérica (Intersex Society of North America), "intersexual" designa una variedad de condiciones en las cuales una persona nace con una anatomía sexual o reproductiva que no se ajusta a las definiciones típicas de masculino o femenino.

${ }^{7} \mathrm{~L} * \mathrm{~s}$ niñ*s intersex son por lo general intervenid*s en forma compulsiva cuando son muy pequeñ ${ }^{*}$ para que sus cuerpos se ajusten a una apariencia masculina o femenina (cfr. Raíces Montero 2010). Por desgracia, sus derechos quedaron fuera de la ley de 2012 y constituyen una deuda pendiente (Maffia 2013). En este trabajo, sólo me referiré a la intersexualidad en la medida en que su existencia constituye, en los términos de la filosofía de la ciencia, una "anomalía", pero los procedimientos médicos a los que son sometid*s $1 *$ s niñ ${ }^{*}$ intersex debe ser objeto de un análisis profundo al que no me dedicaré aquí.
} 
biología en estas cuestiones y, a la vez, acudir a (reglamentar, ampliar el acceso a, e incluso celebrar) ciertas prácticas biomédicas que se basan, en última instancia, en la biología y la bioquímica. Tales prácticas no son independientes de las condiciones de producción del conocimiento teórico que implican ni de los propios compromisos teóricos realistas, reduccionistas, binarios y esencialistas que el conocimiento científico supone. En la mayoría de los debates, el papel de la ciencia se discute desde un punto de vista externo a la ciencia misma - un punto de vista que la filosofía de la ciencia suele considerar externalista- Lo que ocupa el centro de los debates es la función normalizadora de la medicina, los efectos nocivos, los daños sobre los cuerpos y los efectos psicológicos de los tratamientos; desde otras perspectivas, se analizan los efectos sociales y políticos de los tratamientos, haciendo énfasis en que contribuyen a la extensión de los derechos y del reconocimiento. No obstante, también es necesario criticar los supuestos teóricos en el conocimiento biológico y bioquímico sobre los que se erigen tanto los procedimientos quirúrgicos como los tratamientos hormonales, una tarea que la filosofía de la ciencia no puede desconocer.

\section{La comprensión científica de la determinación sexual y sus anomalías}

Como señalé, buena parte de los colectivos militantes cuestionan a la ciencia su derecho a definirnos: lo que ella denomina "dimorfismo sexual" no es determinante de la identidad de género. ¿En qué consiste el dimorfismo sexual científico? ¿Cómo caracteriza la biología esa determinación binaria del sexo? La biomedicina distingue tres o cuatro niveles de diferenciación sexual: 1) el nivel del sexo genético o cromosómico —cromosomas XX o XY-; 2) el nivel del sexo gonadal -ovarios o testículos- y 3) el nivel del sexo genital —vagina, vulva, pene, próstata- determinados en el periodo fetal. Se suele añadir: 4) el nivel del sexo psicosexual y el sexo social (que se determinaría en la pubertad), que incluye los denominados "caracteres sexuales secundarios" (cfr. Maffia 2013).

Si bien la filosofía de la ciencia contemporánea puede considerar en varios sentidos superado el reduccionismo científico, la situación de las ciencias efectivas es otra. En el campo de las propias ciencias biológicas aún impera un reduccionismo que da por sentada la preeminencia epistemológica de la genética y de la biología molecular sobre el resto de las subdisciplinas biológicas (Rosenberg 1997, Rosenberg 2006). Este reduccionismo se erige sobre la base del compromiso ontológico de que todos los aspectos del organismo biológico se reducen

Revista de Filosofía Diánoia, vol. 65, no. 84 (mayo-octubre de 2020) e-ISSN: 1870-4913 • DOI: https://doi.org/10.22201/iifs.18704913e.2020.84.1587 
al (y, por lo tanto, se explican a partir del) plano genético-molecular. En este sentido, si bien la biología distingue entre los niveles mencionados, para los casos "normales" de la diferenciación sexual considera que, en sentido estricto, hay un único criterio determinante del sexo: el genético. La idea es que el nivel de la determinación genética es el que prevalece, el que verdaderamente importa, porque produce la cadena de efectos causales normales que permite determinar los niveles subsidiarios, el gonadal y el genital. Es decir, en última instancia, la determinación del sexo en human*s es cromosómica, depende del denominado "sistema XX-XY". De acuerdo con este nivel de determinación, el sexo se fija en el momento de la fecundación por medio de la composición cromosómica del individu*, lo que la ciencia explica del siguiente modo: las células humanas se dividen en células somáticas, que son diploides, es decir, contienen dos series (dos juegos) de cromosomas, y células reproductivas —óvulos y espermatozoides-, que son haploides, esto es, contienen un solo juego de cada cromosoma. Todas las células somáticas en hembras humanas contienen dos cromosomas sexuales $\mathrm{X}$ (XX), pues las hembras sólo producen este tipo de cromosomas. Los machos, en cambio, producen tanto cromosomas sexuales $\mathrm{X}$ como $\mathrm{Y}$, $\mathrm{y}$ todas sus células somáticas contienen un cromosoma $\mathrm{X}$ y un cromosoma Y (XY). Durante la fertilización, dos células haploides se combinan y forman una célula diploide, con dos pares de cromosomas. Según cuál sea el cromosoma en el gameto masculino, que es el único caso en que puede variar, la célula fecundada tendrá el genotipo XX o el genotipo XY, lo que determina el sexo del embrión (femenino y masculino, respectivamente). La determinación del sexo en human*s se explica, entonces, simplemente en términos de la presencia o ausencia del cromosoma Y. Se considera que la masculinidad en humanos se determina por la presencia del gen SRY en el cromosoma Y, gen responsable de la formación de testículos.

En sentido estricto, para la biología la determinación del sexo es cromosómica porque en los casos considerados "normales" el sexo genético causa, determina, el sexo gonadal, que a su vez causa el sexo genital/fenotípico. De acuerdo con la ciencia, en estos casos los tres niveles que se distinguen coinciden (femenino es $\mathrm{XX}$, con ovarios, vagina y vulva; masculino es XY, con testículos, pene y próstata). Ahora bien, la distinción entre niveles parece resultar de extrema utilidad para pensar los casos "raros", los casos ilegibles o las anomalías científicas. ¿Por qué? Los fenómenos que se conocen en la actualidad como casos de intersexualidad ponen en cuestión la mencionada "armonía" entre los niveles, es decir, el reduccionismo genético. Se suele distinguir entre diferentes 
tipos de intersexualidad, lo que antes se denominaba "hermafroditismo" y hoy "trastornos del desarrollo sexual" ("DSD" por la expresión en inglés, "Disorders of Sex Development"). Esta última denominación se propuso en el ámbito de la pediatría para designar desarrollos sexuales atípicos y pretende abarcar los distintos tipos de intersexualidad. Considerados "trastornos", estos casos se definen como "condiciones congénitas en las cuales el desarrollo cromosómico, gonadal o del sexo anatómico es atípico" (Hughes, Houk, Ahmed y Lee 2005, p. 554; la traducción es mía). ${ }^{8}$ Así, se encuadran en esta denominación los casos de personas que no presentan concordancia entre los tres niveles de la determinación sexual; por ejemplo, personas que son $\mathrm{XX}$, tienen ovarios y tienen genitales "de apariencia masculina" y personas que son $\mathrm{XY}$ y tienen genitales "de apariencia femenina" o "ambiguos". Entre estas últimas se consideran más frecuentes las personas que presentan distintos tipos de SIA (Síndrome de Insensibilidad a los Andrógenos). También hay personas que presentan tanto tejido ovárico como testicular, y existe también la intersexualidad cromosómica: personas que son $\mathrm{X} 0$-que tienen sólo un cromosoma sexual X, el único tipo de monosomía viable - o personas que presentan tres cromosomas sexuales, $\mathrm{XXY}$ o XXX. En muchos de estos casos que se catalogan como "anormales" la causa se considera conocida y, en otros, la causa es indeterminada.

Hay entonces casos en los que no se da de manera "normal" la "cadena causal" que va del plano cromosómico al gonadal y al genital. Cuando esta cadena no tiene lugar, aunque la ciencia lo considere fundamental, el sexo genético no es el aspecto que se privilegia al intervenir a 1*s niñ"s intersex. En general, ante los casos de infantes intersex, 1*s médic*s, en diálogo con (o a partir de la demanda de) madres y padres, con la intervención de los comités de ética de los hospitales y de acuerdo con ciertos protocolos, deciden optar por un sexo sobre la base de las características fenotípicas (del sexo genital) y dejan de lado el sexo genético. Aquí estamos frente un problema, ante un fenómeno curioso $-\mathrm{y}$ sin duda sugerente para la filosofía de la ciencia y el popular reduccionismo científico-: cuando hay armonía entre los tres niveles, el criterio que cuenta es sólo uno, el genético; pero en los casos en los que no hay armonía, la corrección se realiza sobre la base del sexo genital - considerado, desde un punto de vista teórico, secundario, subsidiario del genético-. La práctica biomédica parece realzar para ciertos casos un reduccionismo genital (reducir la determinación sexual al plano fe-

\footnotetext{
${ }^{8}$ Sobre la denominación DSD y las distintas clasificaciones y nomenclatura en pediatría, véase Hughes, Houk, Ahmed y Lee 2005.
} 
notípico de los genitales), que entra en conflicto con el reduccionismo genético imperante en biología. Y así como se suele cuestionar el reduccionismo genital porque violenta las infancias intersex, del mismo modo la propia existencia de la intersexualidad tiene que poner en entredicho el reduccionismo genético, debe obligar a revisarlo.

Por otra parte, dado el reduccionismo genético que impera en conjunción con un realismo científico ingenuo que sigue vigente entre científic*s y médic*s, se acepta que hay disciplinas (o subdisciplinas) fundamentales que reflejan la realidad tal cual es, que ofrecen una descripción objetiva de las entidades en sí mismas —de las entidades autoidentificantes - y disciplinas (o subdisciplinas) que estudian realidades derivadas, aparentes o secundarias, menos reales que las que estudian las ciencias fundamentales. En este sentido, respecto de la diferenciación sexual resulta elocuente la referencia a un cuarto nivel, el nivel psicosexual de la determinación del sexo. Se suele aceptar que hay personas que no se identifican ni con el género masculino ni con el femenino, pero se adjudica esta posibilidad de "ambigüedad" al nivel psicológico y social. La ciencia sostiene que, por el contrario, en el nivel molecular las cosas son de un modo u otro, son así o no son: $\mathrm{XX}$ o XY, con lo que se oculta la existencia de la intersexualidad en el nivel cromosómico.

Muchos colectivos militantes piden a nuestra cultura el reconocimiento de la existencia de la intersexualidad, y apelan a este fenómeno para exigir que la sociedad, que acostumbra arraigar con ahínco en la ciencia occidental muchas de sus creencias morales, deje de dividir el sexo y el género en dos polos antagónicos, masculino y femenino. Así, con independencia de su frecuencia - se suele afirmar que no hay cifras oficiales ni precisas porque, de la mano de la corrección quirúrgica, nuestra sociedad oculta la existencia de personas intersexuales- 9 el fenómeno de la intersexualidad pone en cuestión el binarismo científico que excluye a muchísimas personas. Por otra parte, se cuestiona con fuerza que las correcciones que se hacen a niñ*s intersex se realizan sobre la base del coito heterosexual: se opera para que la persona pueda tener relaciones heterosexuales, sin importar que pueda sentir placer (Maffia 2015, Maffia y Cabral 2003), con lo que se reproduce no sólo el binarismo sexogenérico, sino también el dogma moral de que la sexualidad es heterosexual. Por ello, la intersexualidad como anomalía, es decir, como contraevidencia empírica, caso no esperado dada la "ver-

${ }^{9}$ De acuerdo con la Sociedad Intersex de Norteamérica, una de cada cien personas no se ajusta a los estándares de lo femenino o masculino, y una o dos de mil personas son intervenidas quirúrgicamente para "normalizar" sus genitales. 
dad" de las hipótesis científicas sobre el dimorfismo sexual, debería al menos ser ocasión para revisar los principios teóricos aceptados. ${ }^{10}$

La filosofía general de la ciencia y las filosofías de las ciencias especiales tienen aquí un campo importante de análisis: deben discutir los fundamentos teóricos de las disciplinas, de la biología (evaluar las relaciones entre los distintos niveles de la determinación sexual y dejar un lugar a la intersexualidad) y de la bioquímica (evaluar los fundamentos teóricos en la base de las clasificaciones que se realizan a partir de las características de las hormonas).

La lucha contra la ciencia y sus determinaciones en el campo de las militancias puede encontrar también argumentos en su favor en la filosofía de la ciencia. ¿De qué manera puede esta disciplina contribuir al desmantelamiento de la supuesta determinación de lo científicamente fundado en las vidas sexuales e identidades de género, como históricamente demandan los activismos de las disidencias sexogenéricas? Al postular el dimorfismo sexual, la ciencia legitima un ordenamiento nor$\mathrm{mal} / \mathrm{sano} /$ típico. Si la filosofía de la ciencia puede hacer efectiva su crítica a lo primero, queda al descubierto que la normalización de ese ordenamiento pierde su supuesto sustento. Por otra parte, el enfoque del reduccionismo interteórico constituye un tópico clásico de la filosofía de la ciencia del siglo Xx. Según sostuve, hay un reduccionismo genético en el nivel teórico que entra en conflicto con el reduccionismo genital que parece guiar la práctica médica. Si atendemos ambos reduccionismos, encontramos dos definiciones distintas de lo típicamente masculino y lo típicamente femenino. La filosofía de la ciencia es la disciplina adecuada para hacer patente esta tensión e intentar dar cuenta de ella. ¿Cómo podría hacerlo? En primer lugar, las filosofías especiales de las ciencias deberían incorporar a las discusiones sobre el reduccionismo interteórico el trabajo de explicitar los supuestos teóricos y compromisos ontológicos sobre los que descansan ambos reduccionismos. En segundo lugar, la filosofía de la ciencia debe discutir en relación con el dimorfismo sexual el papel que cumplen los valores que por lo común se consideran extracientíficos, tanto en la práctica de la ciencia y sus aplicaciones como en la misma elaboración de sus productos, las teorías científicas.

\footnotetext{
${ }^{10}$ También se ha desarrollado en la ciencia el denominado "paradigma hormonal" o la "compresión bioquímica" de los sexos. Algunos autores cuestionan el hecho de que dicho paradigma depende mucho de las exigencias de la industria y de los laboratorios (Gaudillière 2004). No me detendré en este debate, pues me alejaría de los propósitos de mi trabajo. Para conocer la discusión, cfr. Sengoopta 2006; para un análisis detallado de los mecanismos hormonales, cfr. Gilbert 2006.
} 
Buena parte de estas tareas las han cumplido filósofas feministas de la ciencia que se han dedicado a desenmascarar las justificaciones de las desigualdades sociales a partir de las diferencias biológicas. También han denunciado que la visión científica del dimorfismo sexual es, al menos, sesgada, y han puesto en cuestión la existencia misma de un método científico, así como la propia noción de objetividad sobre la base de una crítica feminista (cfr. Harding 1992, Harding 1995, Fox Keller y Longino 1996, Longino 1990, Maffia 2005). La filosofía hegemónica de la ciencia tiene aún mucho camino que recorrer para asimilar esta crítica.

Por último, es necesario tener en cuenta, como sostienen las feministas, que el conocimiento científico está lejos de describir el mundo tal cual es, de descubrir el "núcleo natural" del sexo. El conocimiento científico se erige sobre compromisos y valores que son extracientíficos. Debemos indagar hasta qué punto impera aún un realismo esencialista y un reduccionismo científico que confluyen en la adopción de un binarismo de los sexos (que se pretende que se traduzca en un binarismo de género) sobre la base de compromisos ontológicos que no exigen necesariamente los "resultados científicos".

Este binarismo ¿está en la base de los tratamientos a los que se someten las personas trans cuando pretenden adecuar sus cuerpos a la "masculinidad" o a la "femineidad"? La ciencia a la que acuden, ¿es la misma ciencia que 1*s patologiza, que desconoce el cuarto nivel del sexo (el psicosocial) o supone que depende y es efecto de los otros tres? La ciencia a la que se acude para adecuar los cuerpos a la identidad de género, ¿es una ciencia reduccionista que nunca reconocerá que una persona trans es masculina o femenina porque así lo desea? ¿Continuará la ciencia arrogándose el derecho a definir los sexos de acuerdo con los cromosomas, las gónadas y los genitales? ¿Es posible que la ciencia reconozca que un hombre trans es simplemente un hombre y una mujer trans simplemente una mujer? ¿Es posible pensar en términos filosóficos la identidad sin negar las tensiones que surgen de considerar el papel de la ciencia detrás de las prácticas reguladas por la ley sin dejar de reconocer en serio el deseo de las personas trans?

Si una persona trans decide someterse de manera libre a una operación quirúrgica, ¿se somete necesariamente a adquirir las características típicas de un varón o de una mujer porque los tratamientos médicos no ofrecen lugar a terrenos intermedios para habitar los géneros? Si bien los tratamientos médicos tienden por lo general a una normalización, de la mano de la ley argentina abren opciones nuevas, como argumentaré más adelante.

Revista de Filosofía Diánoia, vol. 65, no. 84 (mayo-octubre de 2020) e-ISSN: 1870-4913 • DOI: https://doi.org/10.22201/iifs.18704913e.2020.84.1587 
4. ¿Cómo puede ser la identidad de género?

Cuando se trata de sexo y de género, el lugar de la ciencia resulta cuestionable porque ésta supuestamente porta una "verdad" que violenta, niega existencias, las excluye y las silencia. Poner en cuestión los propios supuestos teóricos sobre los cuales el conocimiento científico se erige es un modo importante de contribuir con una crítica genuina. Debemos comprender que toda vez que las hipótesis científicas clasifican, definen, corrigen y normalizan, lo hacen sobre la base de muchos compromisos que exceden por completo los límites objetivos de sus casos confirmatorios.

Ahora bien, si tenemos en cuenta las posibilidades que la ley argentina habilita, ¿la ciencia sólo reprime y patologiza la identidad de género? Propongo pensar la identidad de género a partir de ciertas discusiones en el debate feminista y trans; en primer lugar, a partir de la idea de identidad como transformación de Bornstein 1994; en segundo lugar, siguiendo a Heyes 2003, sugiero que la identidad de género es relacional; por último, y dado que la ciencia ocupa sin duda un lugar en las transformaciones posibles y en el sistema relacional del género en que estamos inmers*s, propongo pensar la identidad de género también como biotecnológicamente producida, científicamente gestionada.

\subsection{La identidad es transformativa}

Las identidades trans, pensadas a la luz de los cambios — de DNI y de los cuerpos a través de la medicina- que la ley argentina hace posible, pueden interpretarse como identidades transformativas, como el deseo de convertirse en otro, en otra, en otre, de acuerdo con Kate Bornstein.

$\mathrm{Si}$ se decide modificar el DNI, sólo cabe optar por ahora por "masculino" o "femenino", con lo que quedan fuera otras formas posibles de autopercibirse. Si bien esto puede considerarse aún un pendiente de la ley, tampoco debe negarse que ella contribuye a que sean más vivibles las vidas de muchas personas que, en conflicto con lo que señalan sus documentos, se perciben a sí mismas como mujeres u hombres. Puede argumentarse, junto con Butler 2006, que una vida vivible requiere varios grados de estabilidad, y que el ajustarse a lo masculino o lo femenino puede ser en ocasiones una de las estabilidades que hacen que nuestras vidas sean más tolerables.

Sin embargo, Butler 2006 también sostiene que, si las categorías en las que debemos encasillarnos constituyen restricciones no llevaderas, no es vivible una existencia que debe forzarse a ser encuadrada en ellas. Ahora bien, si afirmamos que la identidad es transformativa, 
ello puede contribuir a la posibilidad de legislar para que se reconozcan otras identidades, otras transformaciones posibles, no binarias. Las personas trans y sus identidades no deben pensarse necesariamente en términos de la transición de femenino-a-masculino o de masculino-afemenino, pues justo la existencia de esas identidades ponen en cuestión los géneros. En este sentido puede leerse la ley ya que, como señala Blas Radi, "aunque la ley no tenía por objetivo desmontar el binario, de hecho sí lo hace y enloquece sus categorías" (Radi 2018). De hecho, en noviembre de 2018, por primera vez en Argentina y en América Latina, se permitió por medio de un fallo judicial la modificación de una partida de nacimiento y el DNI para que no se consigne en ellos el sexo, ni masculino ni femenino, si una persona no se identifica con ninguno de esos géneros (Radi 2018).

Según Bornstein, existen tantas experiencias verdaderas del género como personas que creen que tienen género y más importante que el punto de vista de cada persona es el hecho de que por fin los géneros comiencen a cuestionarse. El deseo transexual no debe necesariamente comprenderse como adaptarse a ciertas categorías identitarias ya fijadas y establecidas: "Para mí, el deseo es las ganas de experimentar alguien o algo que nunca he experimentado, o que no estoy experimentando actualmente" (Bornstein 1994, p. 39; la traducción es mía). La búsqueda de la identidad puede entenderse como un ejercicio de transformación que interpreta el deseo mismo como una actividad transformadora.

Así, podemos comprender las identidades trans como transformativas, pero también podemos pensar de este modo las identidades "ambiguas" y las "fluidas":

Si la ambigüedad es una negativa a entrar en un código de género prescrito, la fluidez es la negativa a permanecer en un género o en otro. La fluidez de género es la capacidad de convertirse de manera libre y consciente en uno o varios géneros de un número ilimitado de géneros, durante cualquier periodo de tiempo y a cualquier ritmo de cambio. La fluidez de género no reconoce límites o reglas de género. Una identidad fluida es, por cierto, una forma de resolver los problemas que implican los límites. Dado que la identidad de una persona cambia, también lo hacen las fronteras y los límites individuales. iEs difícil cruzar un límite que se mueve! (Bornstein 1994, pp. 51-52; la traducción es mía)

Si la identidad es transformativa, entonces es esencialmente mudable, lo que implica una radical puesta en cuestión de los planteamientos filosóficos tradicionales, ya que carece de sentido la postulación de —o la 
pretensión de hallar - un fundamento último que garantice estabilidad. Por último, la idea de identidad como transformación podría postularse no sólo para las identidades trans, fluidas o ambiguas, sino para todas las identidades generizadas, incluso para las identidades cis, pues, ¿qué es ser un hombre? ¿Qué sienten los hombres? ¿Qué es ser una mujer? ¿Cómo se siente una mujer?

\subsection{La identidad es relacional}

Tal como la define la ley argentina, ¿la identidad de género fundada en la autopercepción implica necesariamente que es un producto individual? ¿Que se puede referir a una forma de autenticidad, a un núcleo duro o fuero íntimo de 1*s individu*s? Si aceptamos que la identidad es transformativa, ¿las transformaciones son elegidas y llevadas a cabo por un* sujet* aislad* y libre, cuya individualidad exalta esta idea?

Heyes critica la defensa ingenua de una suerte de libertad de expresión del género. De hecho, cuestiona que Bornstein, a partir de su autobiografía, exalta el "voluntarismo" del género y hace depender la identidad de género en forma exclusiva del deseo propio de 1*s individu*s, de su autopercepción. Pero el género, según Heyes, no puede pensarse como la expresión de un sí mismo individual aislado; por el contrario, es relacional, está inserto en sistemas de opresión: "no se comprende mejor el género simplemente como un atributo de los individuos, sino como un conjunto de relaciones usualmente jerárquicas entre sujet*s diferentemente generizad*s" (Heyes 2003, p. 1094).

A propósito de la ley argentina, podría cuestionarse que exalta $-\mathrm{O}$ supone de manera ingenua- la autonomía y autodeterminación del género, y por lo tanto ensalza una idea acrítica de libertad individual. Si bien la crítica es atendible, también puede matizarse. ${ }^{11}$ Considero no sólo que la visión de Heyes puede compatibilizarse con la de Bornstein y con la ley argentina, sino que es necesario poner en diálogo estas posiciones. Que las identidades de género sean transformativas no implica

${ }^{11}$ La militancia trans, de la que la ley es fruto, no se caracteriza en absoluto por la apelación a la voluntad individual, ni deja jamás de pensar y denunciar las relaciones sociales que oprimen las vidas de las personas trans. Estas personas tampoco dejan de reconocer el papel constitutivo para sus identidades de las "familias" elegidas que permiten sostener sus vidas cuando son expulsadas de sus hogares, de las instituciones educativas y del mundo del trabajo (cfr. Berkins 2015). Por otra parte, tampoco parece razonable exigir que una ley trastoque todas las costumbres y valores de la sociedad que pretende legislar: el DNI es individual, y que no haya terceros que puedan intervenir en su cambio, además de una excelente estrategia, es un modo de protección.

Revista de Filosofía Diánoia, vol. 65, no. 84 (mayo-octubre de 2020) e-ISSN: 1870-4913 • DOI: https://doi.org/10.22201/iifs.18704913e.2020.84.1587 
que no sean relacionales, y la inversa tampoco vale. Es en las relaciones de género - que, como afirma Segato 2003, son relaciones jerárquicas y de poder- donde tiene lugar el deseo de transformación. No hay posibilidad de autopercepción ni de reconocimiento sino dentro un sistema relacional.

Las identidades individuales no deben leerse como si se configuraran por fuera del sistema opresivo que es el género, pues no hay tal cosa como habitar los géneros fuera de dicho sistema. Si fuera posible habitar géneros fuera de los sistemas de opresión, no existiría la violencia a la que las identidades trans son sometidas y no necesitaríamos leyes para protegerlas. Es en el marco de las relaciones de género que las identidades se definen, transforman y ponen en cuestión, y en ese contexto surgen tensiones irresolubles; por ejemplo, ¿̇cuánto puede haber de deseo personal y cuánto de determinaciones ineludibles en un sistema opresor? El papel de la ciencia da cuenta también de esta tensión: transformarse para ser reconocid* en ocasiones implica dolorosamente ser violentamente intervenid* por el sistema médico, pues las relaciones de opresión son relaciones violentas. ${ }^{12}$ Los modos en que nos constituimos todas las personas como personas generizadas no puede pensarse por fuera de la violencia biotecnológica.

Por último, si bien es cierto que, en general, cuando hablamos de binarismo nos referimos al par de opuestos masculino/femenino - de acuerdo con el dimorfismo sexual científico y con las "opciones" en el DNI-, las relaciones de género se comprenden mejor si tomamos el binarismo sujeto universal (público)/sujetos minorizados, parciales (privado), tal como sostiene Segato 2018a. De acuerdo con la autora:

[E]se binarismo determina la existencia de un universo cuyas verdades son dotadas de valor universal e interés general y cuya enunciación es la figura masculina, y sus otros, concebidos como dotados de importancia particular, marginal, minoritaria. El hiato inconmensurable entre lo universalizado y central, por un lado, y lo residual minorizado, por el otro, configura una estructura binaria opresiva y, por lo tanto, inherentemente violenta. (Segato 2018a, p. 221; cursivas en el original)

Este binarismo da cuenta en particular de las relaciones de género y permite comprender las identidades trans como identidades minorizadas en cuanto que están en desacato con respecto al patriarcado, en la medida en que "desafía[n] el orden, la jerarquía patriarcal" (Segato

${ }^{12}$ Véase el informe sobre la violencia a la que son sometidas las travestis, transgéneros y transexuales en Argentina en Berkins 2015.

Revista de Filosofía Diánoia, vol. 65, no. 84 (mayo-octubre de 2020) e-ISSN: 1870-4913 • DOI: https://doi.org/10.22201/iifs.18704913e.2020.84.1587 
2018b, p. 20). Esas identidades desacatadas, ¿logran un reconocimiento genuino o el patriarcado simplemente "resuelve" incorporarlas $-\mathrm{y}$ replicarse a sí mismo- por medio de las intervenciones médicas? ¿Puede la ciencia ayudar a ampliar las libertades? O ¿sólo somete más a las identidades trans? En el mismo sentido, ¿puede la ley contribuir a ampliar las libertades? Más adelante argumentaré que, en lugar de volver a encasillar y de reeducar los géneros, la ley argentina abre el espacio para un reconocimiento genuino. ¿Qué potencia tienen, entonces, la ley y la ciencia juntas?

\section{3. La identidad es biotecnológicamente producida}

Las prácticas biotecnológicas disciplinan y normalizan los cuerpos; por lo tanto, la gestión biomédica de la identidad de género es violenta, como lo son las relaciones de género a partir de las cuales se configuran todas las identidades. Sin embargo, la Ley de Identidad de Género constituye una importante ampliación de los derechos.

Entonces, ¿qué lugar es justo reconocerles a las prácticas médicas $\mathrm{y}$, en última instancia, a los supuestos teóricos en los que se basan? Sin desatender las críticas, debe reconocerse que las biotecnologías y el conocimiento científico que las fundamenta producen identidad, la gestionan. ${ }^{13}$ Si la ciencia contribuye en la producción de la identidad de género por medio de las biotecnologías, es necesario aceptar la tensión que estas técnicas expresan en la medida en que ocupan un lugar productor y transformativo, a la vez que se basan sobre hipótesis binarias. Estos supuestos teóricos no describen una realidad dada, pues no le corresponde a la ciencia descubrir el núcleo natural del género, sino que produce verdades en el nivel teórico que cimientan las prácticas biomédicas. Pero la ley permite que, a su vez, las prácticas biomédicas contribuyan a producir identidades generizadas, no necesariamente binarias,

${ }^{13}$ Las ideas de que las identidades son transformativas y que las producen las biotecnologías, que el género no se ancla en características fijas, así como el reconocimiento de la fluidez del género no deben interpretarse en modo alguno como razones en favor del reconocimiento de un papel productor de identidad a las terapias reparativas. Cabe señalar que, en Argentina, las terapias de reorientación de identidad $\mathrm{u}$ orientación sexual están prohibidas por la Ley Nacional de Salud Mental (Ley 22.914) del año 2010 -otro avance en materia de derechos que debe celebrarse-. En este sentido, debe destacarse también que, en las Normas de Atención para la Salud de Personas Trans y con Variabilidad de Género (WPATH), se señala en forma explícita que las personas trans y con variabilidad de género no son personas enfermas. Véase la séptima versión, de 2012, disponible en <https://www.wpath.org/media/cms/Documents/SOC\%20v7/SOC\%20V7_Spani sh.pdf $>$, consultado el 25/07/2019.

Revista de Filosofía Diánoia, vol. 65, no. 84 (mayo-octubre de 2020) e-ISSN: 1870-4913 • DOI: https://doi.org/10.22201/iifs.18704913e.2020.84.1587 
pues permite la constitución de identidades en total desajuste con los supuestos teóricos que las componen. Si pensamos que las biotecnologías tienen este papel, es posible potenciar el carácter transformativo y dar cuenta del carácter relacional de las identidades.

Respecto del carácter transformativo de la identidad, no cabe duda de que los tratamientos y procedimientos médicos pueden interpretarse como medios productores de identidad porque el acceso a ellos habilita transformaciones en los cuerpos. En lo que se refiere a las posibilidades que abre la ley, al no supeditar un tratamiento a otro ni obligar a ningún tipo de tratamiento o procedimiento quirúrgico en particular, las transformaciones posibles se multiplican: la ciencia contribuye de este modo a pensar cuerpos en desarmonía con la "cadena causal normal" de fenómenos que van desde el nivel genético al gonadal y al genital. Estos tres niveles pueden desacoplarse porque la ley establece una libertad total respecto de la elección de tratamientos y procedimientos. Las transformaciones posibles apelan, en su riqueza y diversidad, a la ciencia, y lo hacen con la posibilidad de contradecir, de cuestionar, en cierta medida, el dimorfismo sexual binario, la cadena causal de fenómenos "normales" que la ciencia postula y los reduccionismos (genético o genital). Por otra parte, en tanto no se fuerzan esterilizaciones, se pueden producir otros tipos de transformaciones como, por ejemplo, que una persona que no quiera ejercer el rol social de "mujer-madre" pueda, sin embargo, gestar. Con esto, una de las consecuencias de la ley es el cuestionamiento de importantes dogmas morales respecto de cómo deben ser las familias y que pretenden fundamentarse en la "naturaleza" o la "biología".

En cuanto al carácter relacional de la identidad, afirmé que ésta se configura a partir de relaciones jerárquicas y violentas. En ese sistema de relaciones, el lugar de poder que reviste la medicina en nuestra sociedad es innegable. Si aceptamos que el sistema médico nos somete, oprime y normaliza, sería muy ingenuo afirmar que, una vez que se establece la ley de identidad de género, la medicina, por fin, se pone sin titubeos "de nuestro lado". Si bien esto es así desde la perspectiva de la ley, las exigencias de las comunidades trans ponen en cuestión, al menos en cierta medida, el saber-poder del sistema médico. Este cuestionamiento - que se articula con la crítica de los géneros- se inscribe y se efectúa en los cuerpos. Por otra parte, la ley obliga al sistema de salud: 1*s médic*s deberán formarse y los hospitales deberán equiparse para poder ofrecer los tratamientos y realizar los procedimientos quirúrgicos que se soliciten. Por supuesto, no debe pensarse que la ley "gana una batalla" contra el poder médico, pero éste debe reconocer,

Revista de Filosofía Diánoia, vol. 65, no. 84 (mayo-octubre de 2020) e-ISSN: 1870-4913 • DOI: https://doi.org/10.22201/iifs.18704913e.2020.84.1587 
valorar y cuidar de estas identidades desacatadas, porque la ley así lo exige. En última instancia, lo que caracteriza el sistema jerárquico relacional del género, es tanto la violencia como el desacato.

\section{Conclusiones}

Por medio de la ley argentina, que obliga al reconocimiento y al trato digno y posibilita el cambio de DNI y el acceso a los tratamientos médicos, se está produciendo un hecho que debe protegerse. No sólo se comienzan a reconocer identidades históricamente violentadas, sino que se está gestionando una identidad de género que debe reconocerse y, por lo tanto, debe protegerse, pues el reconocimiento acarrea un valor: exige protección, ofrece un marco, otorga vivibilidad y habitabilidad en la propia vida y en el mundo. La ley torna público lo que ciertos discursos contrarios a los derechos consideran privado (la autopercepción): no sólo pone de manifiesto y desafía el binarismo masculino/femenino, sino también el binarismo público/privado. Aquello que se produce por medio de la ley y por medio de las transformaciones que ésta posibilita es la identidad de género, ya no como un hecho íntimo, pero tampoco como un hecho "objetivo" a corroborarse o refrendarse, sino como un hecho valuado: la identidad de género autopercibida debe respetarse, reconocerse y protegerse.

Ahora bien, no sería justo enfatizar que las biotecnologías producen identidades de género si esto soslaya el papel que desempeñan los distintos colectivos militantes respecto de la constitución identitaria. En Argentina, los avances respecto del reconocimiento, así como los cambios en los documentos de identidad que comienzan a consignar identidades no binarias — ni varón ni mujer-, son el resultado de la lucha militante. En los últimos años, los movimientos feministas y LGBTTIQ+ han logrado, entre otras cosas, incidir en la agenda parlamentaria. Un ejemplo de esto es el debate por el aborto seguro, legal, gratuito y libre en 2018. Asimismo, la creciente lucha de las comunidades trans introdujo en el debate público la noción jurídica de "transfemicidio" o "travesticidio". Por esa razón, debería señalarse que la posibilidad de la constitución de identidades en desacato al patriarcado se debe, de manera destacada, a estas luchas, de la mano de la ley y las biotecnologías.

La ley, en sí misma puede leerse como una superación de la discusión sobre la relación entre el género y la biología, sobre si hay alguna base "natural" de los géneros. Es cierto que la ley supera todo ese debate porque parece no dejar intersticios para que reingrese la ciencia. Sin embargo, aquí he argumentado que, en las prácticas que la ley habilita, 
la ciencia se inmiscuye y participa en la producción de ese hecho valuado que es la identidad. La ciencia no sólo produce transformaciones "de hombre-a-mujer" o "de mujer-a-hombre" (Bettcher 2007, Bettcher 2014) que permiten alcanzar grados de estabilidad importantes y necesarios para muchas personas, sino que también invoca la multiplicidad de transformaciones que podría producir y a las que la ley abre la puerta. Ya sea que se busquen ciertas estabilizaciones, ya sea que se abra la posibilidad a nuevas transformaciones, siempre que la ciencia interviene no es posible pensar que la vivibilidad que implican unas u otras sea posible sin cierta violencia. Toda definición de 1*s sujet*s generizad*s, parciales, no universales, minorizad*s como mujer, como trans, como "otro del hombre", contiene la mediación de la violencia, y la violencia médica y científica es la responsable de definirn*s y encasillarn*s. Si, como sostiene Butler, "la tecnología es un locus de poder en el cual lo humano es producido y reproducido" (Butler 2006, p. 27), entonces ¿pueden escapar a la violencia la constitución de la identidad de género y nuestras vidas, en general, tan dependientes de las biotecnologías? Quizá en un futuro, si la ciencia nos acompaña (o nos abandona), quizá en ese futuro (muy triste futuro para los negocios médicos) ya no se sea necesario acudir a prácticas biotecnológicas para producir identidades. Pero ese tiempo no ha llegado todavía. ${ }^{14}$

\section{Referencias bibliográficas}

Berkins, Lohana y Josefina Fernández, 2006, La gesta del nombre propio, Madres de Plaza de Mayo, Buenos Aires.

Berkins, Lohana (comp.), 2015, Cumbia, copeteo y lágrimas. Informe nacional sobre la situación de travestis, transexuales y transgéneros, 2a. ed., Madres de Plaza de Mayo, Buenos Aires.

Bettcher, Talia Mae, 2007, "Evil Deceivers and Make-Believers: On Transphobic Violence and the Politics of Illusion", Hypatia, vol. 22, no. 3, pp. 43-65, $<$ https://doi.org/10.1111/j.1527-2001.2007.tb01090.x>.

Bettcher, Talia Mae, 2014, "Feminist Perspectives on Trans Issues", en Edward N. Zalta (comp.), The Stanford Encyclopedia of Philosophy (edición primavera de 2014), disponible en <https://plato.stanford.edu/archives/spr2014/ entries/feminism-trans/>, consultado el 2/9/2018.

Bornstein, Kate, 1994, Gender Outlaw. On Men, Women and the Rest of Us, Routledge, Nueva York, <https://doi.org/10.4324/9780203365991>.

${ }^{14}$ Este trabajo se realizó gracias al apoyo del CONICET y la UBA. La autora agradece a los revisores anónimos, cuyos comentarios y sugerencias enriquecieron el trabajo. 
Butler, Judith, 1990, Gender Trouble. Feminism and the Subversion of Identity, Routledge, Nueva York, <https://doi.org/10.4324/9780203824979>.

Butler, Judith, 2006, Deshacer el género, trad. Patricia Soley-Beltrán, Paidós, Barcelona.

Cabral, Mauro, 2015, "Post Scriptum", en Berkins 2015, pp. 145-149.

Defreyne, Justine, Laurens D.L. van de Bruaene, Ernst Rietzschel, Judith van Schuylenbergh y Guy G.R. T’Sjoen, 2019, "Effects of Gender-Affirming Hormones on Lipid, Metabolic, and Cardiac Surrogate Blood Markers in Transgender Persons", Clinical Chemistry, vol. 65, no. 1, pp. 119-134, <https:// doi.org/10.1373/clinchem.2018.288241>.

Diamond, Milton y Keith Sigmundsen, 1997, "Sex Reassignment at Birth: A Long Term Review and Clinical Implications", Archives of Pediatrics and Adolescent Medicine, no. 151, pp. 298-304, <https://doi.org/10.1001/archpedi. 1997.02170400084015>.

Fausto-Sterling, Anne, 2006, Cuerpos sexuados. La política de género y la construcción de la sexualidad, trad. Ambrosio García Leal, Melusina, Barcelona.

Flores Bedregal, Teresa, 2003, "El género no debería ser una categoría dual”, Rebelión, disponible en <http://www.rebelion.org/noticia.php?id=2876>, consultado el 5/10/2018.

Fox Keller, Evelyn y Helen Longino, 1996, Feminism \& Science, Oxford University Press, Oxford.

Gaudillière, Jean-Paul, 2004, "Genesis and Development of a Biomedical Object: Styles of Thought, Styles of Work and the History of the Sex Steroids", Studies in History and Philosophy of Biological and Medical Sciences, vol. 35, no. 3, pp. 525-543, <https://doi.org/10.1016/j.shpsc.2004.06.003>.

Gilbert, Scott (comp.), 2006, Developmental Biology, 8a. ed., Sinauer, Sunderland, Mass.

Giorgi, Gabriel, 2014, "Genética marcada", en Informe Escaleno, disponible en $<$ http://www.informeescaleno.com.ar/index.php?s=articulos\&id $=269>$.

Harding, Sandra, 1992, "After the Neutrality Ideal; Science, Politics and Strong Objectivity", Social Research, vol. 59, no. 3, pp. 567-587.

Harding, Sandra, 1995, "Strong Objectivity: A Response to the New Objectivity Question", Synthèse, vol. 104, no. 3, pp. 1-19, <https://doi.org/10.1007/ BF01064504>.

Hausman, Berenice, 1995, Changing Sex: Transsexualism, Technology, and the Idea of Gender, Duke University Press, Durham N.C., <https://doi.org/10.12 15/9780822396277>.

Heyes, Cressida, 2003, "Feminist Solidarity after Queer Theory: The Case of Transgender", Signs. Journal of Women in Culture and Society, vol. 28, no. 4, pp. 1093-1120, <https://doi.org/10.1086/343132>.

Heyes, Cressida, 2018, "Identity Politics", en Edward Zalta (comp.), The Stanford Encyclopedia of Philosophy(edición otoño 2018), disponible en $<$ https:// plato.stanford.edu/archives/fall2018/entries/identity-politics/ $>$, consultado el 05/11/2018.

Revista de Filosofía Diánoia, vol. 65, no. 84 (mayo-octubre de 2020) e-ISSN: 1870-4913 • DOI: https://doi.org/10.22201/iifs.18704913e.2020.84.1587 
Hughes, Ieuan A., Christopher Houk, Sied Faisal Ahmed y Peter Allen Lee, 2005, "Consensus Statement on Management of Intersex Disorders", Archives of Disease in Childhood, vol. 91, no. 7, pp. 554-563, <https://doi.org/ 10.1136/adc.2006.098319>.

Instituto Nacional contra la Discriminación, la Xenofobia y el Racismo (INADI), 2016, "Cuatro años de la ley de identidad de género", disponible en $<\mathrm{http} / / /$ www.inadi.gob.ar/2016/05/10/cuatro-anos-de-la-ley-de-identidad-de-gene ro-en-argentina/>, consultado el 20/10/2018.

Khan, Jenna, Robert L. Schmidt, Matthew J. Spittal, Zil Goldstein, Kristi J. Smock y Dina N. Greene, 2019, "Venous Thrombotic Risk in Transgender Women Undergoing Estrogen Therapy: A Systematic Review and Metaanalysis", Clinical Chemistry, vol. 65, no. 1, pp. 57-66 < https://doi.org/10.1373/ clinchem.2018.288316>.

Longino, Helen, 1990, Science as Social Knowledge. Values and Objectivity in Scientific Inquiry, Princeton University Press, Princeton.

Maffia, Diana, 2005, "Conocimiento y emoción", Arbor, vol. 181, no. 716, pp. 516-521, <https://doi.org/10.3989/arbor.2005.i716.408>.

Maffia, Diana, 2013, "Cuerpo para armar (y destrozar)", Página/12, suplemento de Soy, disponible en <https://www.pagina12.com.ar/diario/suplement os/soy/1-2959-2013-05-31.html>, consultado el 10/11/2018.

Maffia, Diana, 2015, "La Ley es un avance extraordinario", en Río Negro, disponible en $<$ https://www.rionegro.com.ar/region/diana-maffia-la-ley-es-un-a vance-extraordinario-DCRN_7983817>, consultado el 10/11/2018.

Maffia, Diana y Mauro Cabral, 2003, "Los sexos ¿son o se hacen?", en Diana Maffia (comp.), Sexualidades migrantes. Género y transgénero, Feminaria, Buenos Aires, pp. 86-96.

Money, John y Richard Green, 1969, Transsexualism and Sex Reassignment, Johns Hopkins University Press, Baltimore.

Mouratian, Pedro, 2012, "Ciudadanía plena para todas y todos", en Instituto Nacional contra la Discriminación, la Xenofobia y el Racismo, Hacia una Ley de Identidad de Género, disponible en <https://www.educ.ar/recursos/121 188/hacia-una-ley-de-identidad-de-genero $>$, consultado el 18/10/2018.

Radi, Blas, 2018, "Caro Gero y su nuevo DNI. Un mundo sin sexo legal", Revista Anfibia, disponible en $<$ http://revistaanfibia.com/ensayo/mundo-sin-sexolegal/>, consultado el 20/11/2018.

Raíces Montero, Jorge Horacio, 2010, "Epistemología de las intersexualidades", en Jorge Horacio Raíces Montero (comp.), Un cuerpo: mil sexos. Intersexualidades, Topia, Buenos Aires, pp. 15-35.

Raymond, Janice G., 1979, The Transsexual Empire. The Making of the She-Male, Beacon Press, Boston.

Rosenberg, Alex, 1997, "Reductionism Redux: Computing the Embryo", Biology and Philosophy, vol. 12, no. 4, pp. 445-470, <https://doi.org/10.1023/A:10 06574719901>.

Rosenberg, Alex, 2006, Darwinian Reductionism. Or, How to Stop Worrying and Love Molecular Biology, University of Chicago Press, Chicago. 
Segato, Rita, 2003, Las estructuras elementales de la violencia. Ensayos sobre género entre la antropología, el psicoanálisis y los derechos humanos, Universidad Nacional de Quilmes, Buenos Aires.

Segato, Rita, 2018a, La guerra contra las mujeres, Prometeo, Buenos Aires.

Segato, Rita, 2018b, Contra-pedagogías de la crueldad, Prometeo, Buenos Aires. Sengoopta, Chandak, 2006, The Most Secret Quintessence of Life. Glands, Sex, and Bodies, 1850-1950, University of Chicago Press, Chicago.

Recibido el 23 de noviembre de 2018; revisado el 27 de julio de 2019; aceptado el 22 de agosto de 2019. 